\title{
$\beta$-Adrenoceptor blockade in sclerosing cholangitis of Mdr2 knockout mice: antifibrotic effects in a model of nonsinusoidal fibrosis
}

\author{
Ingo Strack ${ }^{1,4}$, Sigrid Schulte ${ }^{2,4}$, Heike Varnholt ${ }^{1,3}$, Stephanie Schievenbusch ${ }^{1}$, Ulrich Töx ${ }^{2}$, Katharina Wendland ${ }^{1}$, \\ Hans-Michael Steffen ${ }^{2}$, Uta Drebber ${ }^{1}$, Hans-Peter Dienes ${ }^{1}$ and Margarete Odenthal ${ }^{1}$
}

Primary sclerosing cholangitis (PSC) is a cholestatic liver disease with high propensity to develop into cholangiocarcinoma. The hepatobiliary disorder of PSC is due to progressive fibrosis surrounding the intra- and extrahepatic bile ducts. Until now, no effective medical therapy exists. To study the progression of sclerosing cholangitis after inhibition of the sympathetic nervous system by blockade of the $\beta$-adrenoceptors, we used the $\mathrm{Mdr} 2^{-1-}$ mouse model, which develops periportal fibrosis similar to human PSC. Liver tissues of $\mathrm{Mdr}^{-1-}$ mice untreated or treated with the $\beta$-adrenoceptor antagonist propranolol were analyzed for inflammation and fibrosis progression at different time points by histological scoring and immunostaining for $\alpha$-smooth muscle actin ( $\alpha$-SMA), CD45 and S100A4. Transaminases and hydroxyproline contents were determined. Expression of angiotensinogen, endothelin-1, TGF- $\beta$, TNF- $\alpha$, CTGF and procollagen $1 \mathrm{~A} 1$ was studied by real-time PCR on laser-microdissected areas of acinar zones I and II-III. After 3 months, periportal fibrosis had developed in $\mathrm{Mdr}^{-1-}$ mice, but immunostaining revealed no sinusoidal and only minor periportal contribution of myofibroblasts with prominent fibroblasts. Propranolol treatment of $\mathrm{Mdr}^{-1-}$ mice improved liver architecture.

Additionally, inflammation and fibrosis were significantly reduced. After 3 months of treatment, the antifibrotic effect of the $\beta$-blockade was most obvious. The transcript levels of procollagen 1A1, TNF- $\alpha$, TGF- $\beta$, CTGF and endothelin- 1 were markedly repressed in the portal areas of treated mice. Taken together, these data show that propranolol efficiently delays progression of sclerosing cholangitis. Therefore, the blockade of $\beta$-adrenoceptors is a promising option to support future therapeutic strategies in the treatment of human PSC.

Laboratory Investigation (2011) 91, 252-261; doi:10.1038/labinvest.2010.162; published online 4 October 2010

KEYWORDS: Abcb4; fibroblasts; fibrosis; Mdr2; primary sclerosing cholangitis; propranolol; sympathetic nervous system

Primary sclerosing cholangitis (PSC) is a serious chronic cholestatic liver disease of unknown etiology, ${ }^{1}$ characterized by progressive inflammation, concentric periductal (onion skin-like) obliterative fibrosis of intra- and extrahepatic bile ducts leading to cholestasis, cholangitis and cirrhosis. ${ }^{1}$ In addition to cirrhosis, $10-15 \%$ of the patients develop cholangiocarcinoma, increasing the morbidity and mortality of this disease. ${ }^{2}$ To date, many therapies with different drugs, including monotherapies with, for example, corticosteroids, colchicine, cyclosporine, methotrexate, tacrolimus and ursodeoxycholic acid (UDCA), ${ }^{3-8}$ or combinations of these drugs have been studied. The most promising drug so far has been UDCA, showing beneficial effects primarily on liver biochemistry. ${ }^{9}$ Thus, there is currently no proven pharmacological therapy for PSC. ${ }^{3}$ The most definitive treatment of PSC is still liver transplantation. However, antifibrotic drugs have not yet been tested. Previous studies have shown that inhibition of the renin-angiotensin system (RAS), by both angiotensin-converting enzyme (ACE) inhibitors and angiotensin II receptor 1 (AT-1) antagonists, improve sinusoidal liver fibrosis. ${ }^{4}$ Hence, treatment of rats suffering from fibrosis on $\mathrm{CCl}_{4}$ intoxication or bile duct ligation, both involving hepatic stellate cells (HSCs) as the main producers of extracellular matrix components, resulted in a marked reduction of collagen accumulation. ${ }^{5,6}$ Furthermore, in vitro studies demonstrated that blocking of the angiotensin pathway not

\footnotetext{
${ }^{1}$ Institute for Pathology, University Hospital of Cologne, Cologne, Germany; ${ }^{2}$ Department of Gastroenterology and Hepatology, University Hospital of Cologne, Cologne, Germany and ${ }^{3}$ Department of Pathology and Laboratory Medicine, University of North Carolina at Chapel Hill, Chapel Hill, NC, USA

Correspondence: Dr M Odenthal, PhD, Institute for Pathology, University Hospital of Cologne, Kerpener Strasse 62, 50924 Koeln, Germany. E-mail: m.odenthal@uni-koeln.de

${ }^{4}$ These authors contributed equally to this work. 
only reduces the expression of collagens but also the secretion of the crucial fibrogenic mediator TGF- $\beta .^{7}$ Oben et al demonstrated that the sympathetic nervous system (SNS) also affects collagen synthesis and proliferation of HSCs in vitro ${ }^{8,10}$ and in vivo. ${ }^{11,12}$ Moreover, Dubuisson et al ${ }^{13}$ were able to show that chemical sympathectomy leads to attenuated fibrosis in a $\mathrm{CCl}_{4}$-induced sinusoidal fibrosis model.

In this study, we used the Mdr2 ${ }^{-1-}$ model mimicking PSC and addressed the question whether inhibition of the $\beta$ adrenoceptors also affects fibrosis not involving myofibroblastic HSCs. The applied mouse model is characterized by the disruption of the multidrug resistance gene $2(\mathrm{Mdr} 2)^{14}$ that encodes a canalicular transporter responsible for the secretion of phosphatidylcholine into bile. ${ }^{15}$ The lack of biliary phospholipids secretion in $\mathrm{Mdr}^{-/-}$mice leads to increased biliary concentrations of non-micellar-bound free bile acids. As a result, there is damage of tight junctions and basement membranes of bile ducts, leading to leakage of potentially toxic bile acids into the periductal area, inducing inflammation, onion skin-like periductal fibrosis and intraand extrahepatic strictures and dilatations comparable with the characteristics of human PSC. ${ }^{16,17}$

Here, we demonstrate that propranolol treatment results in significant deceleration of experimental sclerosing cholangitis. Therefore, $\beta$-blockade may be considered as a novel supporting therapeutic strategy for the treatment of nonsinusoidal fibrosis in human PSC.

\section{MATERIALS AND METHODS}

\section{Animals}

$\mathrm{Mdr2}{ }^{-I-}$ knockout mice were kindly provided by F. Lammert (Internal Medicine II, Saarland University Hospital, Homburg/ Saar, Germany). Wild-type control mice were obtained from the animal facility of the Institute for Pathology, University Hospital of Cologne. All mice were bred on Balb/c genetic background. The animals were housed with a 12-h light-dark cycle, water and food consumption was permitted ad libitum.

$\mathrm{Mdr}^{-1-}$ controls $(n=92)$ and wild-type mice $(n=13)$ received a standard mouse diet (Altromin, Lage, Germany). Starting at day 21 after birth, $\operatorname{Mdr} 2^{-1-}$ mice $(n=73)$ received a diet containing propranolol- $\mathrm{HCl}(60 \mathrm{mg} / \mathrm{kg}$ body weight $)$ (Altromin). All three groups consisted of an almost balanced number of male and female animals (Supplementary Table 2).

The animals were killed after $3(n=35), 6(n=28), 12$ $(n=42), 20(n=32)$ and $32(n=32)$ weeks of propranolol or control treatment. All experimental protocols were approved by the local Animal Care and Use Committee (50.302.2-K22, $24 / 05$ ), according to the criteria outlined in the Guide for the Care and Use of Laboratory Animals prepared by the National Academy of Sciences, as published by the National Institutes of Health $(\mathrm{NIH}, 1996) .^{18}$

\section{Blood Pressure Measurement}

Blood pressure of the mice was measured by using the tailcuff method (Harvard Apparatus, Holliston, USA). A random selection of propranolol-treated $(n=9)$ and -untreated mice $(n=12)$ was used, and the average blood pressure from three independent readings was calculated.

\section{Tissue Processing and Measurement of Liver Enzymes}

At the designated time points, the mice were killed by cervical dislocation. Liver samples were snap frozen in liquid nitrogen and stored at $-80^{\circ} \mathrm{C}$ until further use, as well as fixed for $24 \mathrm{~h}$ in $4 \%$ neutral-buffered formalin and embedded in paraffin. For this purpose, livers were rapidly excised and two samples, each, were taken from separate lobes.

Additionally, a serum sample of every mouse was collected to determine the alkaline phosphatase (AP), alanine and aspartate aminotransferase (ALT and AST) liver enzyme levels using the Reflotron system (Roche diagnostics, Mannheim, Germany) according to the manufacturer's instructions.

\section{Determination of Hydroxyproline Content}

Hydroxyproline content of the liver was quantified colorimetrically using $100 \mathrm{mg}$ frozen liver tissue samples according to the method of Jamall et al ${ }^{19}$ and the modification of Cho et $a{ }^{20}{ }^{20}$ as described previously. ${ }^{21}$

\section{Histological Evaluation of the Tissues}

Sections of paraffin-embedded liver tissue were prepared and stained with hematoxylin and eosin, Gomori methenamine silver, Sirius red and chloracetate esterase to allow histological evaluation of liver fibrosis (staging) and the inflammatory activity in the liver (grading). The histology of specimens was independently and blindedly assessed by two board-certified pathologists (HV and UD). For all cases, a mean score of both embedded liver fragments was generated. Grading of inflammation and staging of fibrosis were performed according to the four-tiered Batts-Ludwig system. ${ }^{22}$ Furthermore, the extent of bile ductular proliferation was scored using a grading system of $0-3$, as described by Farazi et $a l^{23}$ and CK19 immunostaining.

\section{Immunohistology}

Immunohistochemical stainings of human PSC $(n=18)$ and experimental sclerosing cholangitis $(n=165)$ specimens against $\alpha$-smooth muscle actin (SMA), CD45, F4/80, CK19 and S100A4 were performed according to the ABC method, as described earlier. ${ }^{13}$ The antigen retrieval was carried out by microwave treatment in $\mathrm{pH} 6$ citrate buffer, in case of the F4/ 80 staining by using trypsin, and the antibodies were diluted as follows: $\alpha$-SMA (Sigma-Aldrich, Deisenhofen, Germany) 1:60; CD45 (Abcam, Cambridge, USA) 1:500; F4/80 (Serotec, Duesseldorf, Germany) 1:100; CK19 (Abcam) 1:200; and S100A4 (Dako, Hamburg, Germany) 1:1000.

\section{RNA Isolation and Reverse Transcription}

Total RNA was isolated from $30 \mathrm{mg}$ snap-frozen liver tissue using the Nucleospin II Kit (Macherey \& Nagel, Düren, Germany). A measure of $1 \mu \mathrm{g}$ of total RNA from each sample 
was reverse transcribed using the $\mathrm{ABI}$ high-capacity kit (Applied Biosystems, Foster City, USA), according to the manufacturer's recommendations. cDNA samples were diluted with tRNA $(50 \mu \mathrm{g} / \mathrm{ml})$ to obtain a final concentration of $10 \mathrm{ng} / \mu \mathrm{l}$. A measure of $1 \mu \mathrm{l}$ was analyzed by real-time PCR.

\section{Laser-Associated Microdissection, RNA Isolation and Reverse Transcription of Microdissected Specimens}

Snap-frozen liver tissue of 3-month-old propranolol-treated $(n=6)$ and control $(n=6) \mathrm{Mdr}^{-1-}$ mice was embedded in Tissue-tek solution (Sakura Finetek, Torrance, USA), cut in $5 \mu \mathrm{m}$-thick sections and stained with hematoxylin. A total of 10 portal fields (acinar zone I) and 10 parenchymal areas (acinar zone II-III) of each liver specimen with a diameter of $200 \mu \mathrm{m}$ were laser microdissected (P.A.L.M., Bernried, Germany). The tissue samples of the portal fields and the samples of the parenchymal areas were pooled and total RNA was isolated using the Nucleospin XS Kit (Macherey \& Nagel), as described by the manufacturer. Reverse transcription of total RNA was carried out by the Superscript III reverse transcriptase enzyme and random hexamer primers from Invitrogen (Karlsruhe, Germany). Undiluted cDNA samples were analyzed in real-time PCR.

\section{Real-Time PCR}

Relative mRNA transcript level were quantified on Stratagene MX3000 Cycler (Stratagene, Cedar Creek, USA) by using 10 ng of cDNA either with SYBR Green Master Mix PCR Kit (Applied Biosystems) or RealMasterMix Probe Kit (Eppendorf, Hamburg, Germany) and Taqman probes according the recommendations of the supplier. All reactions were run in triplicate and normalized to hypoxanthine phosphoribosyl transferase (HPRT), which was used as housekeeping gene. Transcript levels were quantified using a dilution series as
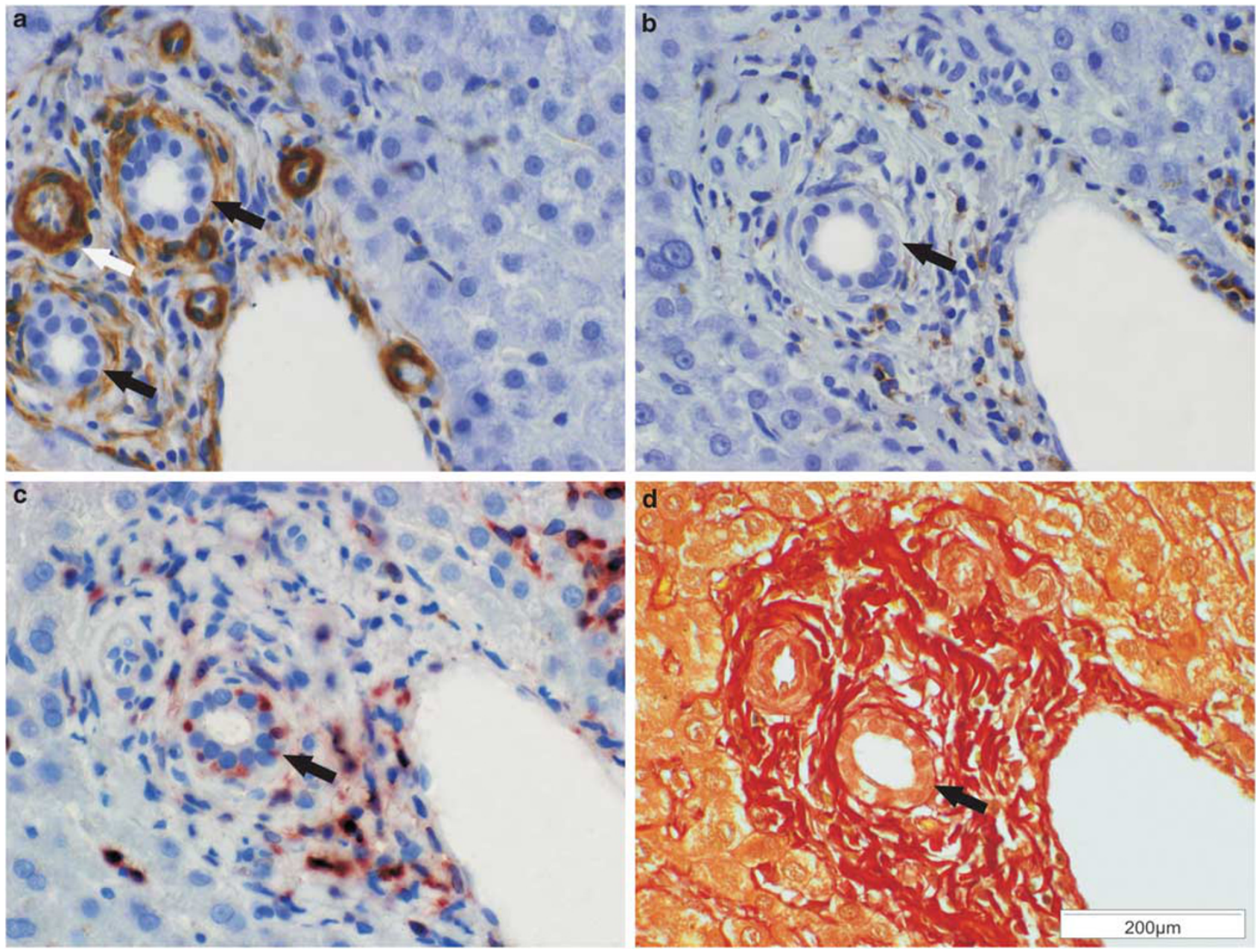

Figure 1 Myofibroblasts and fibroblasts present in the sclerosing portal fields, but not in the sinusoidal interstitium of human PSC. Immunohistochemistry of $\alpha$-SMA (a), CD45 (b) and S100A4 (c), and histochemical Sirius red staining (d) on serial liver sections demonstrates occurrence of $\alpha$-SMA-positive myofibroblasts (a) and infiltration of CD45- (b) and S100A4-positive (c) cells in the sclerosing areas around the injured bile ducts (black arrows). Additionally, $\alpha$-SMA staining indicates vascular smooth muscle cells of the portal arteries and the portal vein (white arrow). The Sirius red staining (d) shows the deposition of collagens around the injured bile ducts. 
standard curve. Primer sequences are summarized in Supplementary Table 1.

\section{Statistical Analysis}

Statistical analysis was performed with SPSS15 Software (SPSS, München, Germany). Data are reported as arithmetic means \pm s.d. Statistic analysis was performed by Student $t$-test when appropriated. $P$-values $<0.05$ were considered as significant.

\section{RESULTS}

Portal Myofibroblasts and Fibroblasts Occur in Human PSC and in Mdr2 ${ }^{-1-}$ Mice on Cholestasis

The characterization of cells contributing to the progression and matrix deposition in human PSC, as shown in Figure 1 by $\alpha$-SMA and S100A4 staining, revealed occurrence of fibroblasts and $\alpha$-SMA-positive myofibroblasts in fibrotic portal areas. Myofibroblasts were primarily located around the bile ducts. However, no myofibroblastic cells were
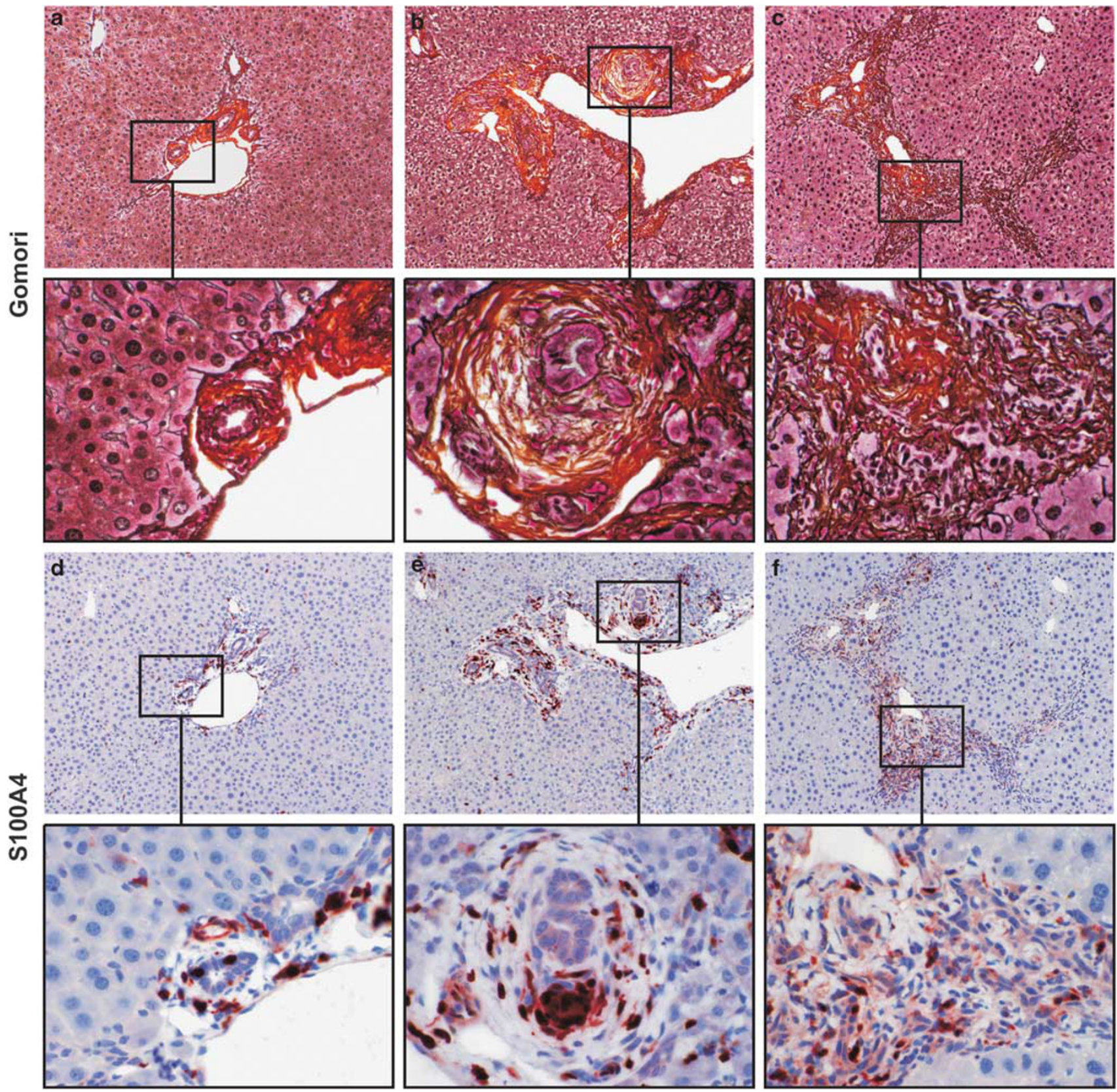

Figure 2 Progression of portal fibrosis in Mdr2 ${ }^{-1-}$ mice. Serial sections of the livers of 6-week, 3-month and 5-month-old Mdr2 ${ }^{-/-}$mice (scale bar represents $200 \mu \mathrm{m}(\mathbf{a}-\mathbf{i})$ and $100 \mu \mathrm{m}$ in the corresponding magnification). Increasing deposition of collagen is shown in the portal areas with a typical onion skin-like pattern (b), leading to the formation of bridges and septa (c) (a-c, Gomori staining). Immunochemical S100A4 staining (d-f) demonstrates prominent recruitment of fibroblasts to the portal areas and the fibrotic septa during progression of fibrosis. $\alpha$-SMA-positive myofibroblasts are restricted to the sclerosing areas around the bile ducts in the early stages, shown by $\alpha$-SMA immunostaining $(\mathbf{g}-\mathbf{i})$. 


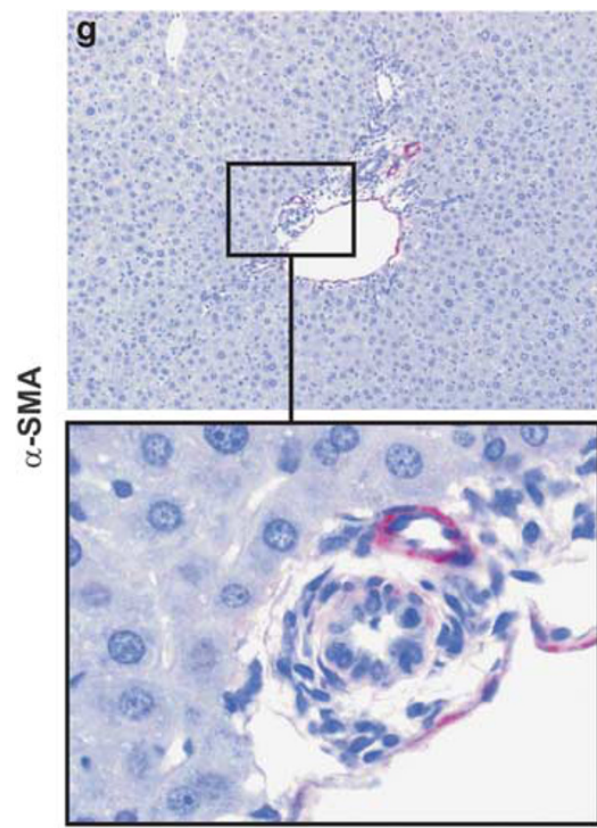

6 weeks

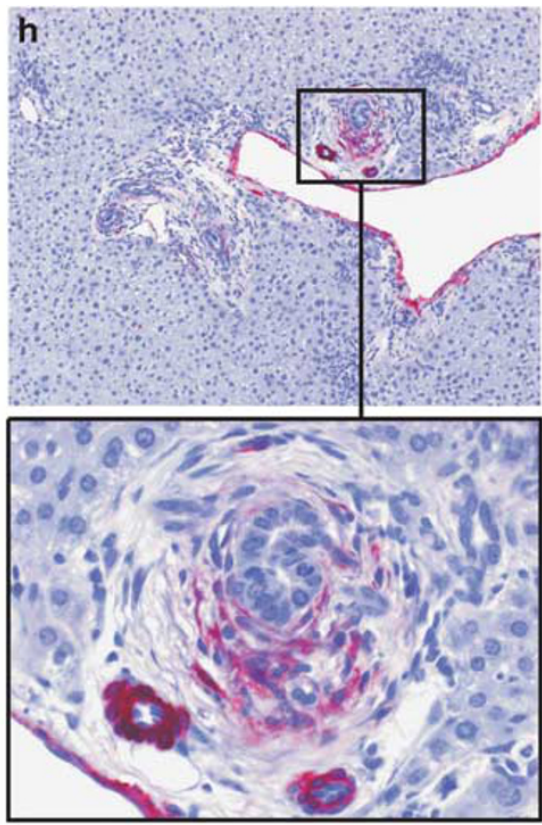

3 months

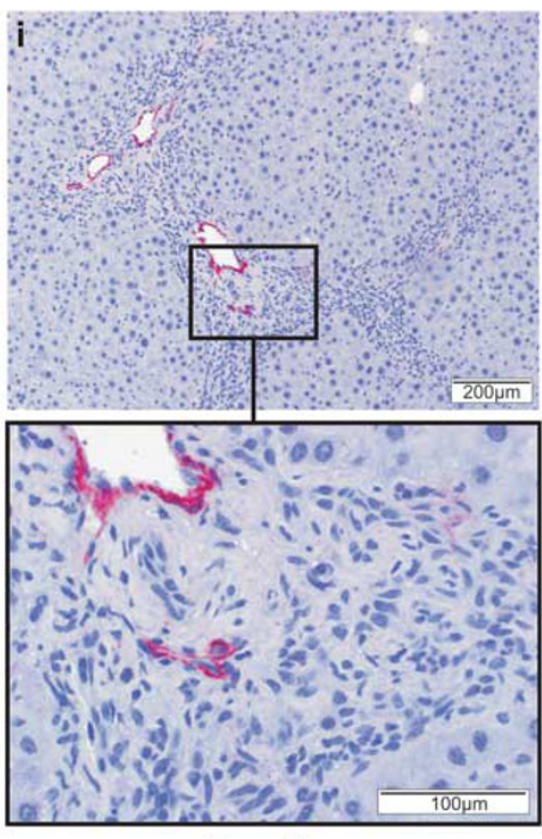

5 months

Figure 2 Continued.

Table 1 Grade of inflammation and stage of fibrosis in $\mathrm{Mdr2}^{-1-}$ mice with or without propranolol treatment

Inflammation

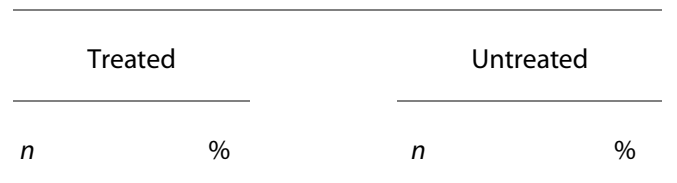

Grade

$\begin{array}{rrcrr}\text { G0 } & 19 & 26.0 & 11 & 12.0 \\ \text { G1 } & 39 & 53.4 & 49 & 53.3 \\ \text { G2 } & 14 & 19.2 & 25 & 27.2 \\ \text { G3 } & 1 & 1.4 & 7 & 7.6 \\ \text { Total } & 73 & 100.0 & 92 & 100.0 \\ & & P \text {-value: } 0.029 & & \end{array}$

Fibrosis

\begin{tabular}{rrrrr}
$\begin{array}{rrrr}\text { Stage } \\
\text { S0 }\end{array}$ & 10 & 13.7 & 5 & 5.4 \\
S1 & 25 & 34.2 & 24 & 26.1 \\
S2 & 32 & 43.8 & 40 & 43.5 \\
S3 & 5 & 6.8 & 21 & 22.8 \\
S4 & 1 & 1.4 & 2 & 2.2 \\
Total & 73 & 100.0 & 92 & 100.0 \\
& & P-value: 0.024 & & \\
\hline
\end{tabular}

observed in the lobule (Figure 1a). CD45 staining (Figure 1b) suggests that some of S100A4 (Figure 1c)-positive cells originate from circulating fibrocytes derived from the bone marrow and characterized by both S100A4 and CD45 positivity. ${ }^{14}$

To evaluate the effect of propranolol treatment on sclerosing cholangitis, we used the Mdr2 $2^{-1-}$ mouse model and compared first the cellular pattern involved in fibrosis of $\mathrm{Mdr} 2^{-/-}$mice with the distribution of fibrogenic cells of human PSC.

$\mathrm{Mdr} 2^{-1-}$ mice developed fibrosis within 3 months, but at the age of 6 weeks, matrix deposition restricted to the immediate vicinity of the bile ducts was already observed (Figure 2a). At the age of 3 months, the periportal fibrosis was characterized by the typical onion skin-like matrix formation around the bile ducts (Figure 2b). After 5 months, septal formation and massive matrix deposition was shown (Figure 2c). In agreement with human PSC, myofibroblasts of $\mathrm{Mdr} 2^{-l-}$ livers were restricted to the portal fields (Figure $2 \mathrm{~g}-\mathrm{i}$ ), and no activated myofibroblasts were observed in the parenchyma. Numerous S100A4-positive fibroblasts occurred periductally and also in the septae when bridging fibrosis had developed (Figure 2d-f). Corresponding to the data in human PSC, a portion of these S100S4-positive cells were also CD45 positive (Supplementary Figure 1).

This data demonstrates that the Mdr2 ${ }^{-1-}$ model reflecting the cellular pattern of human PSC was suitable for the evaluation of propranolol treatment.

\section{Blockade of the $\beta$-Adrenoceptors Resulted in Deceleration of PSC-Associated Fibrosis}

Beginning at the age of 3 weeks and extending to different time points, $\mathrm{Mdr} 2^{-1-}$ mice were treated with propranolol. 
No differences were noticed in behavior and development of animals that were treated with propranolol. Long-termtreated Mdr2 $2^{-1-}$ mice show a minor reduction in the average
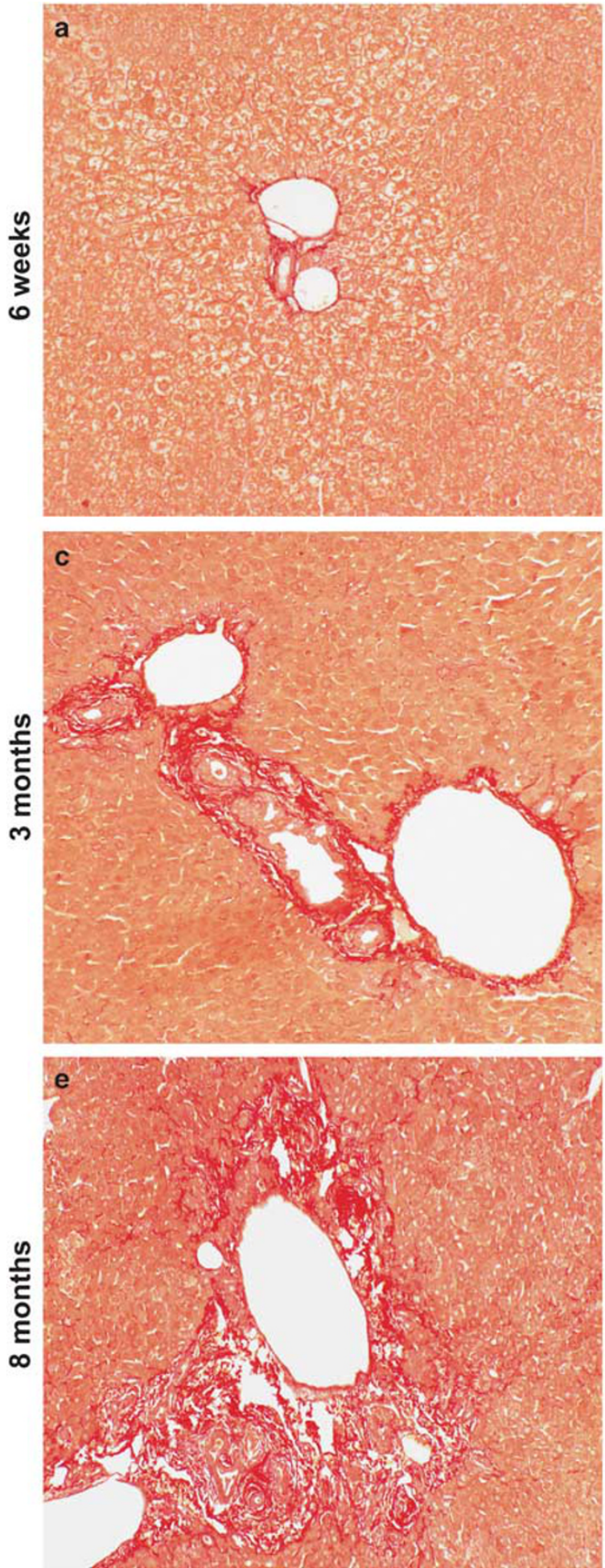

treated systolic blood pressure $(81.2 \pm 7.9$ versus $91.8 \pm 12.1 \mathrm{~mm} \mathrm{Hg}$; $P<0.05$; Supplementary Figure 2) without an impact on the viability. The histological assessment of bile duct prolifera-
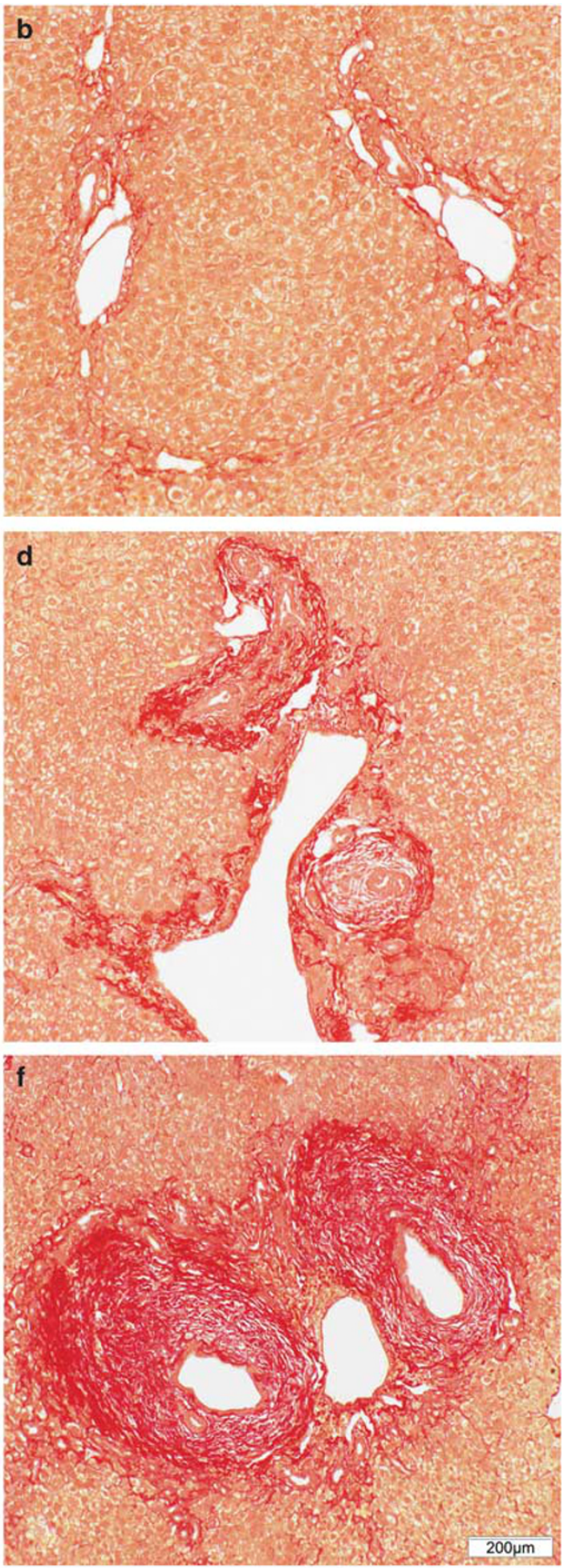

untreated

Figure 3 Deceleration of portal fibrosis in $\mathrm{Mdr}^{-1-}$ mice after propranolol treatment. Sirius Red staining of representative liver sections from propranololtreated (a, c, e) and -untreated (b, d, f) Mdr2 ${ }^{-1-}$ mice after 6 weeks $(\mathbf{a}, \mathbf{b}), 3$ months $(\mathbf{c}, \mathbf{d})$ and 8 months (e, f) of treatment. The staining demonstrates less collagen deposition in the portal area and lesser expansion of fibrosis, especially in the early stages, in propranolol-treated Mdr ${ }^{-/-}$mice. 
tion and CK-19 immunostaining (Supplementary Figure 4) did not reveal a significant difference between treated and untreated $\mathrm{Mdr} 2^{-/-}$mice. However, investigation of macrophage infiltration by F4/80 immunostaining (Supplementary Figure 5) demonstrated a significant beneficial impact of propranolol treatment on the grade of inflammation (Table 1). Additionally, 6 weeks of treatment of $\mathrm{Mdr}^{-1-}$ mice resulted in an obvious restriction of fibrosis to the portal areas (Figure 3a), while periportal fibrosis had already begun to develop in untreated mice (Figure 3b). At the age of 3 months, most of the mice exhibited features of periportal fibrosis, but the matrix deposition and the extension of the onion skin-like periductal sclerosis was less pronounced in the treated mice (Figure 3c) compared with the controls (Figure 3d). After 8 months, in both groups periportal fibrosis was frequently accompanied by septae formation and prominent proliferation of the bile ducts (Figure $3 e$ and $\mathrm{f}$ ). Although the propranolol effect was diminished, the positive effect of the $\beta$-blockade was still noticed after 8 months by slightly but significantly improved liver architecture.

Overall, the propranolol-treated mice showed a significant reduction of fibrosis progression (Table 1). However, differences in biochemical analyses of ALT, AST and AP were small (data not shown) and hydroxyproline values were moderately improved by propranolol treatment (Supplementary Figure 3A). Similarly, analyses of collagen expression (Supplementary Figure $3 \mathrm{~B}$ ) as well as the analyses of TIMP-1 (Supplementary
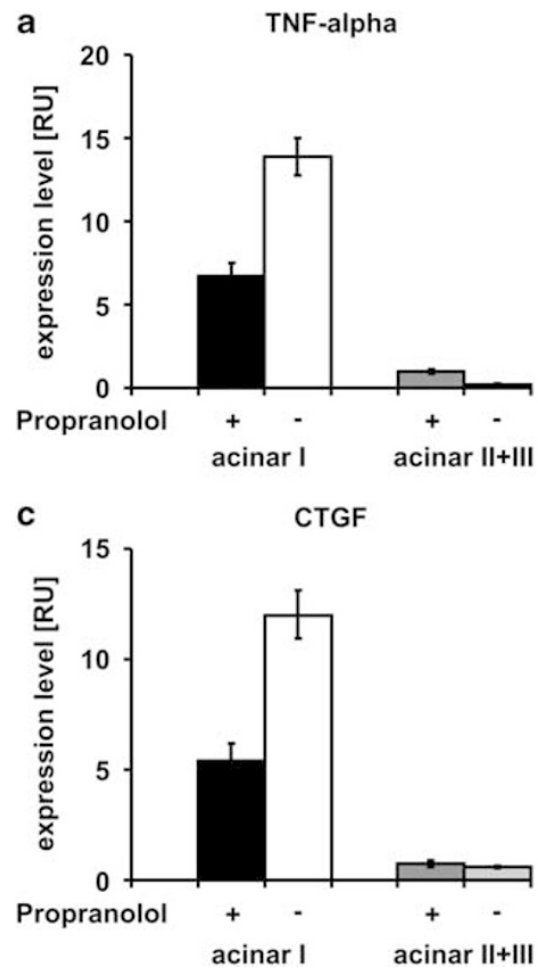

Figure 6) revealed no significant alteration on transcript levels. In addition, the expression of the profibrotic mediator TGF- $\beta$ (Supplementary Figure 3C) showed no difference between treated and untreated $\mathrm{Mdr}^{-l-}$ mice. Therefore, we decided to perform laser microdissection of the liver tissue in order to analyze the affected portal areas selectively.

\section{Propranolol Treatment Effects Periportal Expression of Profibrogenic Marker Expression}

With assistance of the laser microdissection technology, it was possible to analyze expression of profibrogenic markers in portal areas in comparison with the nonfibrotic lobule.

By analyses of RNA extracted from microdissected areas, we showed that the mRNA levels of TNF- $\alpha$, TGF- $\beta$, CTGF and procollagen $1 \mathrm{~A} 1$ were markedly downregulated in the portal areas of propranolol-treated animals. Profibrogenic expression of TNA- $\alpha$, TGF- $\beta$ and CTGF was reduced by $>50 \%$ in fibrotic portal areas. However, the expression of TGF- $\beta$, CTGF and procollagen 1A1 in the lobule was not involved in sclerosing cholangitis and showed no differences after propranolol treatment (Figure 4).

\section{Propranolol Treatment Results in an Altered Expression Pattern of Proinflammatory Mediators Involved also in Vasoconstriction}

We analyzed the effect of propranolol treatment on the expression levels of proinflammatory mediators that exhibit
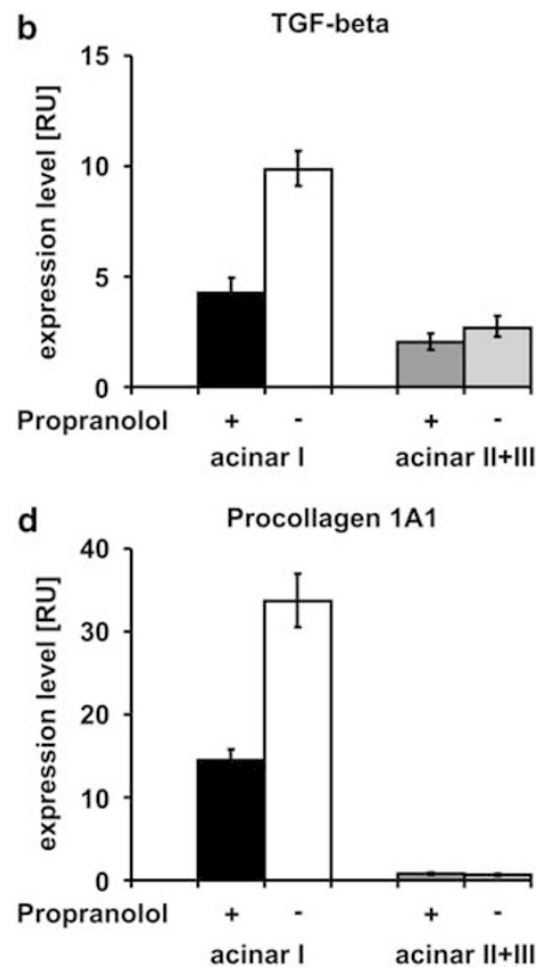

Figure 4 Expression of TNF- $\alpha$, TGF- $\beta$, CTGF and procollagen 1A1 in the portal area (acinar zone I) versus the lobule (acinar zone II-III) of treated and untreated MDR2 ${ }^{-1-}$ livers. Liver sections, obtained from six treated and six untreated $\mathrm{Mdr}^{-1-}$ mice, were laser microdissected, and from each specimen 10 acinar zone I $(n=60)$ and 10 acinar zones II-III samples $(n=60)$ were used for RNA isolation. Transcript levels of TNF- $\alpha(\mathbf{a})$, TGF- $\beta$ (b), CTGF (c) and the procollagen $1 \mathrm{~A} 1$ subunit (d) were analyzed by quantitative real-time PCR. 
a

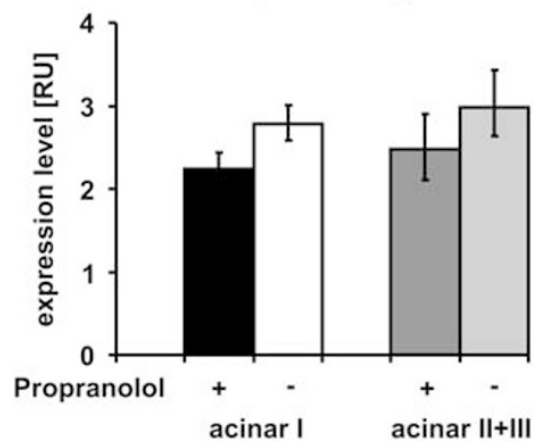

C

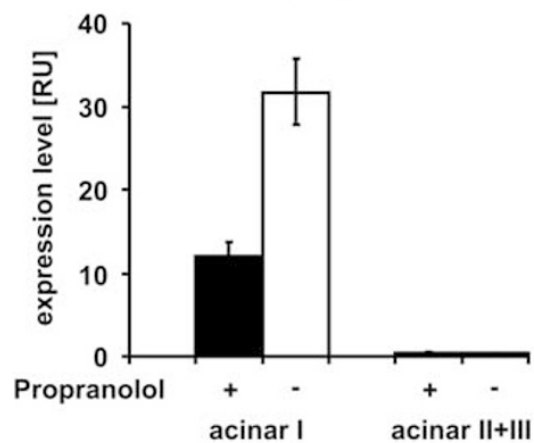

b

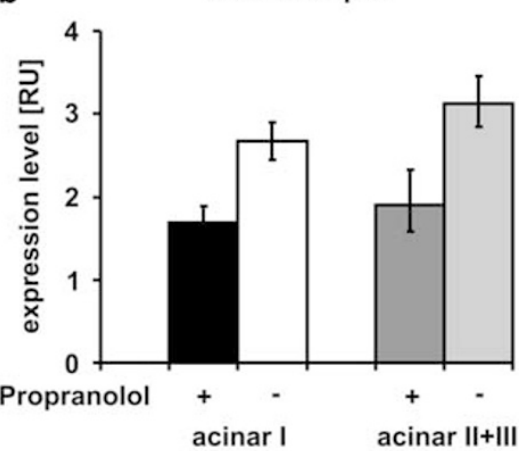

d

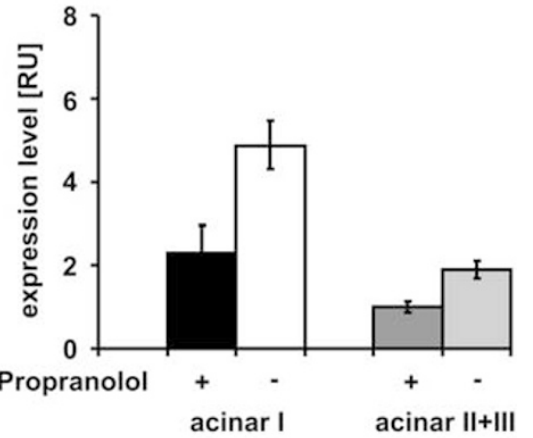

e

Endothelin Receptor B

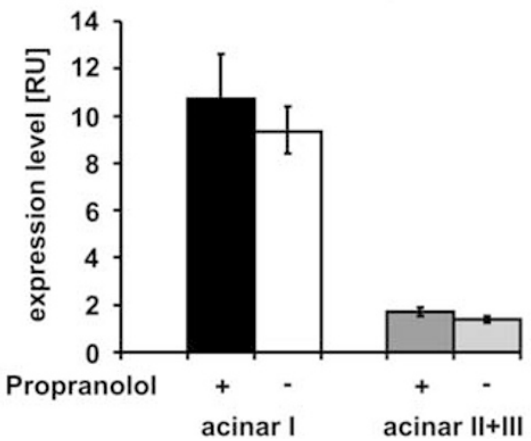

Figure 5 Expression of profibrogenic and vasoconstricive mediators in the acinar zone I versus zone II-III of treated and untreated MDR2 ${ }^{-1-}$ mice. Transcript levels of angiotensinogen (a) and endothelin-1 (c), as well as their receptors AT-1 (b), endothelin receptor A (d) and endothelin receptor B (e) were analyzed in the laser-microdissected portal areas (acinar I, $n=60$ ) and lobules (acinar II-III, $n=60$ ) from propranolol-treated and -untreated MDR2 ${ }^{-1-}$ livers.

additional vasoconstrictive properties under the SNS control. Angiotensinogen transcript levels were reduced by $\sim 17 \%$ in the portal areas and $19 \%$ in the lobule parenchyma in treated mice, whereas the mRNA of the AT-1 showed a reduction of 36 and 39\%, respectively. The mRNA expression of both, endothelin receptors (ET-A, ET-B) and endothelin-1, were higher in portal areas than in the parenchyma. Whereas ET-A receptor transcription levels were reduced by $\sim 50 \%$ after propranolol treatment, propranolol seems to have only a moderate effect on the ET-B receptor transcription. The transcript levels of ET-B receptor were increased by $14 \%$. The most pronounced effect of the propranolol treatment was found in changes of endothelin-1 expression levels.
Endothelin-1 mRNA levels were reduced by $66 \%$ in the portal areas, whereas the mRNA expression in the parenchyma showed no differences between treated and untreated mice (Figure 5).

\section{DISCUSSION}

The results of this study demonstrated that propranolol treatment leads to evident amelioration of fibrosis and inflammation in $\mathrm{Mdr}^{-1-}$ mice. Previous in vitro experiments of Oben et $a l^{10}$ had shown that HSCs that are crucial for sinusoidal fibrosis producing most of the interstitial $\mathrm{ECM}^{24}$ express $\alpha$ - and $\beta$-adrenoceptors. Additionally, HSCs are able to biosynthesize catecholamines because of their 
subset of metabolizing enzymes. ${ }^{10}$ The inhibition of fibrogenic features of HSCs by blockade of the adrenoceptors was supported by studies of Dubuisson et al. These authors demonstrated that sympathectomy after 6-hydroxydopamine application leads to $60 \%$ reduction of fibrosis after CCL4 intoxication. ${ }^{25}$ Our studies using the $\mathrm{Mdr} 2^{-1-}$ model now proves that the blockade of $\beta$-adrenoceptors by propranolol results in a significant deceleration of fibrosis progression. Thus, our data add to the evidence that delaying the fibrotic process because of $\beta$-blockade is not only a matter of inhibition of the fibrogenic properties of HSC but also a more general phenomenon.

In contrast to portal fibrotic lesions due to chronic viral or other cholestatic liver diseases, in which portal myofibroblasts are the main fibrogenic cell types, ${ }^{26,27}$ in both human PSC and experimental sclerosing cholangitis, only small numbers of periportal myofibroblasts are present. Myofibroblasts of PSC are mainly distributed in the immediate vicinity of sclerosing areas around the bile ducts. Thus, it is a point of discussion whether these myofibroblasts originate from cholangiocytes that have undergone epithelial-tomesenchymal transition. ${ }^{28-30}$

However, S100A4 immunostainings demonstrated numerous fibroblasts that appear in the portal field and in the developing septae. Additionally, previous studies have demonstrated that S100A4- and CD45-positive fibrocytes, derived from bone marrow, infiltrate the injured liver. ${ }^{21,28}$ However, scattered occurrence in the periportal area leads to the assumption that they participate in fibrosis, but to a much lower extent than CD45-negative but S100A4-positive fibroblasts. ${ }^{31}$

The limited periportal accumulation of fibrogenic cells in early sclerosing cholangitis might explain why an inhibition of fibrosis cannot be recognized by molecular analyses of the whole liver. Fink et $a l^{32}$ already pointed out that differentiation of cells of injured and noninjured areas significantly improves molecular analyses. Thus, our molecular analyses of the laser-microdissected portal and periportal areas revealed a remarkable downregulation of procollagen $1 \mathrm{~A} 1$ transcripts and of profibrogenic mediators such as TGF- $\beta$, CTGF and TNF- $\alpha$. We propose an inhibition of the fibrogenic properties in fibroblasts involved in sclerosing cholangitis because fibroblasts of different tissues were shown to express the whole subset of $\beta$-adrenoceptors. ${ }^{33,34}$ Therefore, fibroblasts may be directly affected by the inhibition of $\beta$-adrenoceptors in a manner similar to that recently described for HSC by Oben et al. ${ }^{10}$ As vagotomy has been shown to reduce bile flow, whereas elevated levels of neuropeptide $\mathrm{Y}$ increase the bile flow, improvement after $\beta$-adrenoceptor inhibition could also be a matter of altered bile composition or reduced bile flow. ${ }^{35}$ In addition, an indirect effect on fibrosis is also suggested because the RAS is highly controlled by the SNS. Paizis et al have reported that angiotensinogen, renin and ACE expression is strongly upregulated during fibrogenesis, ${ }^{26,27}$ and high systemic levels of angiotensin II have been shown to exacerbate liver fibrosis. ${ }^{26}$ Here, we demonstrate that propranolol treatment leads to a decreased angiotensinogen expression in experimental sclerosing cholangitis. As processing of angiotensinogen into the vasoactive and profibrotic angiotensin II form depends on renin, which in turn is rigorously controlled by the SNS, reduced angiotensinogen levels are suggested to be the indirect response to the $\beta$-blockade. A central role of the angiotensin pathways can also be inferred from a recent review by Toex et al who summarized published data on diminished fibrosis by RAS intervention after administration of ACE inhibitors or AT-1 receptor antagonists with ensuing decrease in TGF- $\beta$ synthesis. ${ }^{4}$ However, additional interaction sites of the blockade of the $\beta$-adrenoceptors have to be considered, because the combined treatment of $\mathrm{Mdr}^{-/-}$mice with both the AT-1 receptor antagonist telmisartan and propranolol improved each of the single therapeutical approaches. ${ }^{36}$

Thus, the expression of endothelin-1, another vasoactive and profibrotic mediator, was decreased by $66 \%$ in the portal areas of propranolol-treated $\mathrm{Mdr} 2^{-1-}$ mice in a similar manner as the proinflammatory and profibrotic growth factors TNF- $\alpha$ and TGF- $\beta$. Endothelin-1 expression was shown to depend on angiotensin II stimulation via the ERKsignaling pathway in smooth muscle cells. ${ }^{37}$ As not only endothelin-1 expression is markedly reduced in the sclerosing cholangitis of $\mathrm{Mdr}^{-1-}$ mice but also the expression of the endothelin receptor ET-A, the blockade of $\beta$-adrenoceptors highly effects endothelin-1 signaling. Endothelin-1 is known to act as a proinflammatory and profibrotic factor, inducing CTGF and TGF- $\beta$ secretion. ${ }^{38}$ Thus, impaired expression of endothelin-1 and its receptor ET-A seems to have a high impact on the attenuation of sclerosing cholangitis progression.

Furthermore, PSC patients who suffer from portal hypertension may benefit from the antihypertensive effect of propranolol by reduction of both vasoactive mediators angiotensin II and endothelin-1. Reduced expression of endothelin-1 and angiotensinogen, leading to vasorelaxation, may contribute to a lesser degree of fibrosis and inflammation in treated animals by lowering mechanical stress in the livers of $\mathrm{Mdr}^{-1-}$ mice. Therefore, improvement of experimental sclerosing cholangitis after $\beta$-blockade is based on reduced synthesis of connective tissue, not only due to repression of profibrogenic factors such as TGF- $\beta$ and CTGF but also due to repression of vasoconstricting factors such as endothelin-1 and angiotensin II. This concerted action of nonselective $\beta$-blockade probably provides a new opportunity to support pharmacotherapeutic approaches to human PSC.

Supplementary Information accompanies the paper on the Laboratory Investigation website (http://www.laboratoryinvestigation.org)

\section{ACKNOWLEDGEMENTS}

For excellent technical support, we thank Melanie Ruhrlaender. This study was supported by a Research and Development (R\&D) grant of the medical faculty of the University Cologne (to H-PD). Ingo Strack held a graduate fellowship funded from the University of Cologne. 


\section{DISCLOSURE/CONFLICT OF INTEREST}

The authors declare no conflict of interest.

1. Silveira MG, Lindor KD. Primary sclerosing cholangitis. Can J Gastroenterol 2008;22:689-698.

2. Mendes FD, Lindor KD. Primary sclerosing cholangitis. Clin Liver Dis 2004:8:195-211.

3. Gordon FD. Primary sclerosing cholangitis. Surg Clin North Am 2008;88:1385-1407, x.

4. Tox U, Steffen HM. Impact of inhibitors of the renin-angiotensinaldosterone system on liver fibrosis and portal hypertension. Curr Med Chem 2006;13:3649-3661.

5. Karimian G, Mohammadi-Karakani A, Sotoudeh M, et al. Attenuation of hepatic fibrosis through captopril and enalapril in the livers of bile duct ligated rats. Biomed Pharmacother 2008;62:312-316.

6. Wei YH, Jun L, Qiang CJ. Effect of losartan, an angiotensin II antagonist, on hepatic fibrosis induced by CCl4 in rats. Dig Dis Sci 2004;49: 1589-1594.

7. Kamada $Y$, Tamura $S$, Kiso $S$, et al. Angiotensin II stimulates the nuclear translocation of Smad2 and induces PAI-1 mRNA in rat hepatic stellate cells. Hepatol Res 2003;25:296-305.

8. Oben JA, Roskams $\mathrm{T}$, Yang $\mathrm{S}$, et al. Hepatic fibrogenesis requires sympathetic neurotransmitters. Gut 2004;53:438-445.

9. Maggs JR, Chapman RW. An update on primary sclerosing cholangitis. Curr Opin Gastroenterol 2008;24:377-383.

10. Oben JA, Diehl AM. Sympathetic nervous system regulation of liver repair. Anat Rec A Discov Mol Cell Evol Biol 2004;280:874-883.

11. Oben JA, Roskams $T$, Yang $S$, et al. Sympathetic nervous system inhibition increases hepatic progenitors and reduces liver injury. Hepatology 2003;38:664-673.

12. Oben JA, Roskams $T$, Yang $S$, et al. Norepinephrine induces hepatic fibrogenesis in leptin deficient ob/ob mice. Biochem Biophys Res Commun 2003;308:284-292.

13. Schulte $\mathrm{S}$, Oidtmann $\mathrm{A}$, Kociok $\mathrm{N}$, et al. Hepatocyte expression of angiotensin II type 1 receptor is downregulated in advanced human liver fibrosis. Liver Int 2009;29:384-391.

14. Kisseleva $\mathrm{T}$, Uchinami $\mathrm{H}$, Feirt $\mathrm{N}$, et al. Bone marrow-derived fibrocytes participate in pathogenesis of liver fibrosis. J Hepatol 2006;45:429-438.

15. Elferink RP, Tytgat GN, Groen AK. Hepatic canalicular membrane 1: the role of mdr2 P-glycoprotein in hepatobiliary lipid transport. FASEB 1997:11:19-28.

16. Fickert $P$, Fuchsbichler $A$, Wagner $M$, et al. Regurgitation of bile acids from leaky bile ducts causes sclerosing cholangitis in Mdr2 (Abcb4) knockout mice. Gastroenterology 2004;127:261-274.

17. Lammert F, Wang DQ, Hillebrandt S, et al. Spontaneous cholecystoand hepatolithiasis in Mdr2-/- mice: a model for low phospholipidassociated cholelithiasis. Hepatology 2004:39:117-128.

18. NIH. Guide for the Care and Use of Laboratory Animals. National Academy Press: National Academy of Science, 1996, ISBN: 0-309-05377-3.

19. Jamall IS, Finelli VN, Que Hee SS. A simple method to determine nanogram levels of 4-hydroxyproline in biological tissues. Anal Biochem 1981;112:70-75.
20. Cho JJ, Hocher $B$, Herbst $H$, et al. An oral endothelin-A receptor antagonist blocks collagen synthesis and deposition in advanced rat liver fibrosis. Gastroenterology 2000;118:1169-1178.

21. Kisseleva T, Brenner DA. Fibrogenesis of parenchymal organs. Proc Am Thorac Soc 2008:5:338-342.

22. Goodman ZD. Grading and staging systems for inflammation and fibrosis in chronic liver diseases. J Hepatol 2007:47:598-607.

23. Farazi PA, Zeisberg M, Glickman J, et al. Chronic bile duct injury associated with fibrotic matrix microenvironment provokes cholangiocarcinoma in p53-deficient mice. Cancer Res 2006;66: 6622-6627.

24. Jiao J, Friedman SL, Aloman C. Hepatic fibrosis. Curr Opin Gastroenterol 2009;25:223-229.

25. Dubuisson L, Desmouliere A, Decourt B, et al. Inhibition of rat liver fibrogenesis through noradrenergic antagonism. Hepatology 2002 35:325-331.

26. Bataller R, Gabele E, Parsons $\mathrm{CJ}$, et al. Systemic infusion of angiotensin II exacerbates liver fibrosis in bile duct-ligated rats. Hepatology 2005;41:1046-1055.

27. Paizis G, Cooper ME, Schembri JM, et al. Up-regulation of components of the renin-angiotensin system in the bile duct-ligated rat liver. Gastroenterology 2002;123:1667-1676.

28. Kisseleva T, Brenner DA. Mechanisms of fibrogenesis. Exp Biol Med (Maywood) 2008;233:109-122.

29. Kalluri R, Neilson EG. Epithelial-mesenchymal transition and its implications for fibrosis. J Clin Invest 2003;112:1776-1784.

30. Rygiel KA, Robertson $\mathrm{H}$, Marshall $\mathrm{HL}$, et al. Epithelial-mesenchymal transition contributes to portal tract fibrogenesis during human chronic liver disease. Lab Invest 2008;88:112-123.

31. Roderfeld M, Rath $T$, Voswinckel $R$, et al. Bone marrow transplantation demonstrates medullar origin of CD34+ fibrocytes and ameliorates hepatic fibrosis in Abcb4-/- mice. Hepatology 2010;51:267-276.

32. Fink L, Kwapiszewska G, Wilhelm J, et al. Laser-microdissection for cell type- and compartment-specific analyses on genomic and proteomic level. Exp Toxicol Pathol 2006;57(Suppl 2):25-29.

33. Anesini C, Borda E. Modulatory effect of the adrenergic system upon fibroblast proliferation: participation of beta 3-adrenoceptors. Auton Autacoid Pharmacol 2002;22:177-186.

34. Leicht $\mathrm{M}$, Greipel N, Zimmer H. Comitogenic effect of catecholamines on rat cardiac fibroblasts in culture. Cardiovasc Res 2000;48:274-284.

35. Yoneda $M$, Nakamura K, Yokohama S, et al. Neuropeptide $Y$ stimulates bile secretion via $\mathrm{Y} 1$ receptor in the left dorsal vagal complex in rats. Hepatology 1998;28:670-676.

36. Schulte $\mathrm{S}$, Mende $\mathrm{S}$, Strack I, et al. Combination therapy with telmisartan and propranolol improves liver fibrosis in the MDR2 knockout mouse model. Hepatology 2009;50(Suppl 4):816A.

37. Hong HJ, Chan P, Liu JC, et al. Angiotensin II induces endothelin-1 gene expression via extracellular signal-regulated kinase pathway in rat aortic smooth muscle cells. Cardiovasc Res 2004;61:159-168.

38. Clozel $M$, Salloukh $\mathrm{H}$. Role of endothelin in fibrosis and anti-fibrotic potential of bosentan. Ann Med 2005;37:2-12. 